\title{
Discussion on the Application of Automatic Meter Reading Technology in Coal Mine Power Supply System
}

\author{
Xiaohong Zeng \\ Chongqing College of Electronic Engineering, Chongqing, 401331
}

Keywords: Power. Measurement. Automatic meter reading. Coal mine. Power supply system

\begin{abstract}
Coal mine power supply system is the guarantee of the normal operation of the mine production factors, the power supply system of coal mine production machinery and bearing the daily production of the power required by the foundation. Considering energy saving and environmental protection and rational use of coal resources, automatic meter reading technology has been applied, and played a certain role in the power supply system. Based on the analysis of electric energy meter reading technology, so as to explore its application in coal mine power supply system. In order to prove that, in order to achieve a reasonable power supply, this technique is more suitable for the power supply system of coal mine production.
\end{abstract}

\section{Introduction}

With the improvement in industries, as the basis of industries, resources such as coal have always attracted the attention of government, and a lot of researches have been done to improve the mining efficiency. Although coal mining has experienced a long history in our country, in traditional operation, it mainly relies on the manpower with low efficiency of mining. After the revolution on industries and technology, coal mining has involved in many mechanical equipment, which improves the efficiency of mining. However, according to the on-site surveys, with the improvement on production, the needs from market are increasing, where the supply cannot meet the requirements of desire. From the aspect of the coal mining enterprises, the emphasis of them is to increase the profit. Additionally, in the routine operation, with the participation of mechanical and electrical equipment, the efficiency of production is improved while the consumption of power and petroleum which increases the cost of mining. In recent years, the price of coal has increased, while the cost on human resources is increased accordingly. Therefore, the profit of coal mining enterprises cannot be guaranteed, so that the issue of how to save power consumption has become the significant problem for routine operation of coal mines.

\section{Analysis of Power Measurement Automatic Message Recording Technology}

\section{Definition of Power Measurement Automatic Message Recording Technology}

With the development of science, more and more appliance have participated in our daily life and work environment. With their help, the efficiency of work is improved to a great degree and enriches routine life of people. The use of appliance is followed by the consumption of power and the cost for power generation, no matter the thermal power or hydraulic power generation. Therefore the standard unit for power consumption calculation has been made, and the consumption is recorded by corresponding recorders, to facilitate the power consumptions. In practice, the meter is installed as per users, while the traditional meters are installed on line between the user and the main line; the presented number is the total consumption of power of this user. As the limitation of technology, the calculation of power consumption requires staff to check the recorder periodically and charge the user accordingly. For this mode, it is inefficiency in power consumption calculation for the quantity of recorders is significantly large for staff to check. After years of development, the calculation of power consumption has been improved a lot. For the new technology, it is to install a sensor at the recorder to collect the data from recorder; and then send the data to server via specific transmission mechanism; thereafter the consumption of power will be calculated automatically. When the power 
consumption is reached some set point, the notice will be sent to the user. User can pay the charge via different ways. This method can improve the efficiency on power consumption calculation.

\section{Functions of Power Measurement Automatic Message Recording Technology}

Automatic message recording technology is equipped with distinct characteristics compared with the traditional power consumption calculation method. First is the automation level, which does not require the staff going to check the meters after the installation of the automatic recorder. Second is the accuracy of the calculation. In traditional method, it relies on the manpower to calculate the consumption which always comes with calculation mistakes due to the huge workload; meanwhile, influenced with precision of the meters, the calculation of power consumption is not that accurate. According to analysis, there are two practical methods to realize the automatic message recording. The first one is to install a device with infrared induction on the meter; Staffs are not required to read and record the number from the meter by themselves but receive the data from corresponding receiver. It will guarantee the precision of power consumption calculation, but still require the staff to check the receivers. The second one is to connect all the meters to a server, where the data will be sent from the meter to the server real-time, so that the power consumption will be recorded exactly. The two methods have their own characters respectively, while they can both improve the efficiency in calculation of power consumption compared with the traditional calculation with manpower, particularly for the second automatic recording technology. However, to realize the second method, it will requires the configuration of the meters for the internet module accompanying with the normal calculation function, which will increase the production cost of the meters.

\section{Functions of Power Measurement Automatic Message Recording Technology in Power Supply System for Coal Mines}

\section{Influences on Cost of Production}

In practical production of coal mining, the production cost is the main focus; while among the factors of cost, the consumption of power is a significant factor. On this basis, many mining companies have issued the policies to save the consumption of power in routine operation to the maximum. As the survey to the companies, their policies on saving power consumptions are rather ambiguous, such as cut off the power supply when the mechanical equipment is in rest, for example. Such mode of consumption saving is not scientific or rational. Due to the high consumption of power of coal mining, compared with the normal users, government has divided the charge of power for civil use and for industrial use, in order to control the total consumption of power. While the charge on power for industrial use is higher than that for civil use. Furthermore, with the industries development recently and the increased consumption of power, the power consumption has been calculated in gradient. Therefore, the cost for the coal mining industries with extremely high consumption on power is in sharp rise. Additionally, as the characteristics of power consumption for industry use, the recorders are different with that for civil use. In previous calculation, limited by the precision of meters, the micro-scale consumption of power cannot be detected by the meter. In this case, apart from the huge consumption in production, the small power for lights hasn't been detected which means the error in calculation. After the increase in precision of meters, the mining companies have found even at the same consumption level as before, the record is increased, which is due to the increase precision of meters, and then the cost of their operation is increased as well.

\section{Rational Use of Resources}

Electricity is an important resource, which requires the other resources to generate the power. Especially for the thermal generation, it will consume huge amount of coal. In this case, the wind and hydraulic power generation are the main developing direction in power generation. However, although under the emphasis on replaceable resources, thermal generation is still the main approach for power generation. It is the result of special historical factors, that we do not have advanced economic and technical level. Therefore to save the power consumption, is not only the reduction on mining cost but also the protection of our environment. For the users, it is the basis and significant reference to save power consumption to obtain the power that consumed timely. With the 
understanding of current consumption of power, the industries can make plan to save power consumption for next stage. Therefore, the precise power consumption metering plays a very important role in saving the consumption. During the routine operation of mining, the resources must be allocated rationally to maximum the efficiency of production. As the information construction currently, the equipment in production mostly relies on the power, therefore it is a significant approach to allocate the resources rationally to control the power consumption in coal mining. If the automatic message recording technology is adopted in a mine, the multiple meters can be used in this mine instead of single one previously, and the different meters will calculate the consumption of different part to precise the calculation of total consumption.

\section{Usage of Power Measurement Automatic Message Recording Technology in Power Supply System of Coal Mine}

\section{Control Power Consumption in Coal Mine}

In power supply system of coal mine, the power consumption recorder will be installed between the branch and the main bus of the line. In traditional calculation approach, it relies on manpower to record the data, so that the industries do not know the consumption for that period timely until the recording is finished; meanwhile the manpower recording of the data is in fixed and large interval. In this case, without precise and timely data as reference, it is difficult for the industries to make further and future plan on saving power to reduce their cost. However, after use the automatic message recording technology, the data will be sent to electric power company real-time, and the industries can visit the website to obtain the data. The data is renewed very fast, or even renewed real-time and the industries will master their real consumption of power. According to the real consumption, they can make a targeted plan for power consumption. After the observation on the consumption of power, the plan can be adjusted accordingly, so that the real consumption of power and the cost of production will be reduced.

\section{Consume Electricity Rationally}

In the traditional recording approach, for the convenience to calculate, there is usually one meter for a user. In this way, the industry will know only the total consumption but know nothing about the consumption structure, the consumption for production and that for lighting, for instance. As different appliance has the different influence on the efficiency of production, it will be helpful for the industries to know their specific power consumptions. Therefore they can save the unnecessary power consumption accordingly, optimizing the use of power. The utilization of automatic message recording technology will satisfy the requirements of coal mines. Installing multiple meters as requirements, the computer will calculate the consumption for each part automatically according to the data sent by the meters. Therefore, the mining industries will spend some expense on the installation of these meters to know the specific consumption of the equipment. Furthermore, according to the collected data the use of equipment can be optimized so that improves the use efficiency of power consumption. Therefore, after the application of the new technology, it is significant for both optimization of power consumption and the use of mechanical equipment. After optimization, the consumption of power will be reduced, and the efficiency of production will be improved. The automatic message recording technology has been facilitated widely in western developed countries, while with the limitation of technology, it has not been popularized which has influenced the development of our coal mining industries.

\section{Conclusion}

As the basis of industries development, resources such as coal have always attracted the attention of government. During the ancient time, in order to smelt metals and for the purpose of heating, our country has started mining the coal vastly. However, due to the technology limitation, the efficiency of mining is relatively in low rate. With the revolution of industries, the mechanical equipment has been used in the mining of coal which has increased the efficiency of mining. According to analysis 
of this paper, it has entered the age of information. Power, as the basis of informatization, is the resource for all the electrical equipment to work. The unit of power consumption is the main method to calculate the cost of power consumption which will influence the production cost directly. To use the automatic message recording technology, it is significant to lower down cost of production, to optimize the power consumption structure and to improve the efficiency of production.

\section{References}

[1] Xiao Hua and Wei Zhiquan: Research on Oxygen Adsorption Property in Oxygen Adsorption Detection of Coal Spontaneous Combustion Tendency, Goal Quality and Technology, 2013(01): 37-39

[2] Liu Lijian, Wang Chunhua and Jia Lihong: Typical Problems and Corresponding Solutions for Usage of Coordinate Measuring Machine, Journal of Hebei Industries and Science, 2006(06): 350-352

[3] Yang Changdong: Research of Ventilation and Safety Management in High Gas Coal Mining Projects, Values Engineering, 2013(16): 104-105 